\title{
Cerebral Venous Thrombosis Presenting as Subacute Parkinsonism
}

\author{
Amal Al-Hashmi, Oliver F. Nguyen, Rafael S. Glikstein, Hyman M. Schipper
}

Can. J. Neurol. Sci. 2010; 37: 684-686

Cerebral venous thrombosis (CVT) is an uncommon form of cerebrovascular disease, with an incidence of three to four cases per million persons. Although acute-onset headache is the most consistent presentation, CVT may also manifest as seizures, intracranial hypertension, and focal sensorimotor deficits ${ }^{1}$. Here, we report the unusual development of subacute parkinsonism secondary to multiple cerebral sinus thromboses.

\section{CASE RePORT}

A 57-year-old right-handed woman with a three year history of diffuse, intermittent headaches presented to the Jewish General Hospital (Montreal) with worsening non-pulsatile headache and progressive hypophonia, micrographia, bradyphrenia and gait unsteadiness over a three week period. There were no complaints of hyposmia, sleep disturbances, constipation or other pre-motor symptoms of idiopathic Parkinson disease. Her past medical history was unremarkable and her medication list included vitamin $\mathrm{D}$ and calcium supplements only. Physical examination revealed normal vital signs with no evidence of postural hypotension, normal level of consciousness, orientation, attention, language, affect, judgment and insight. There was facial hypomimia but the rest of the cranial nerve exam, including bilateral olfactory nerve testing, was normal. There was marked hypophonia and micrographia and moderate bradykinesia and cogwheel rigidity. Muscle power was normal and tremor was absent. The gait was narrow-based, small-steppage and unsteady. There was severe retropulsion on pull-testing. Sensory, cerebellar and reflex testing was unremarkable save for prominent glabellar tap and palmomental signs.

A computed tomogram (CT) scan of the head (Figure) showed small, scattered hyperdensities in bilateral frontal, temporal and parietal lobes and in the region of the interpeduncular cistern. The white matter appeared abnormally hypodense consistent with generalized edema (a1-a2). Magnetic resonance imaging axial FLAIR sequences showed heterogeneous hyperintense signal of the transverse sinuses (left, arrow) and congested peripheral veins (b1-b2). Axial FLAIR and T2 sequences showed diffuse, hyperintense signal of the periventricular and subcortical white matter and mild sulcal effacement (c1-c2). Magnetic resonance venography (MRV) demonstrated occlusion of the transverse sinuses and distal internal cerebral veins, minimal flow within the vein of Galen, and severely congested cortical and subcortical veins and straight sinus (d1-d2).

Routine hematology, erythrocyte sedimentation rate and thyroid stimulating hormone were normal and an investigation for hypercoagulable state, including homocysteine, protein $\mathrm{C}$, protein S, lupus anticoagulant, antithrombin III, factor V Leiden and common prothrombin mutations, was unrevealing. Tests for antinuclear antibodies and rheumatoid factor were negative. Work-up for occult malignancy, including CT of the chest, abdomen and pelvis and total body positron emission tomography, was negative. A cerebrospinal fluid examination showed normal opening pressure of $15 \mathrm{~cm} \mathrm{H}_{2} \mathrm{O}$, no cells, normal protein and glucose, and negative bacterial/fungal cultures.

Despite intravenous heparin and a therapeutic partial thromboplastin time, there was abrupt clinical deterioration characterized by confusion, worsening gait and double incontinence. A repeat CT of the head was unchanged. A trial of intra-jugular tPA and mechanical venous sinus thrombectomy were unsuccessful due to "rock-hard" clot consolidation. The patient was started on warfarin and dexamethasone with gradual improvement of neurological status to near-admission levels. She was discharged to a rehabilitation hospital on warfarin. An outpatient re-evaluation two months after discharge from the Jewish General Hospital disclosed dramatic improvement in her clinical status with only minimal unsteadiness on tandem gait and slowing of finger movements remaining. A repeat MRI/MRV scan performed approximately one month later revealed marked improvement of periventricular and subcortical white matter hyperintensities, but no significant interval change in the extensive thrombosis of the cerebral veins.

\section{Discussion}

This middle-aged woman presented with a three month history of worsening headaches and three weeks of progressive parkinsonism characterized by rigidity, bradykinesia, gait and postural instability, bradyphrenia, hypophonia, and micrographia. Neuroimaging revealed severe, multiple cerebral vein and sinus thromboses as the cause of the extrapyramidal syndrome.

From the Department of Neurology (AA-H, OFN, HMS), Radiology (RSG), Jewish General Hospital and McGill University, Montreal, Quebec, Canada.

Received February 22, 2010. Final Revisions Submitted April 8, 2010.

Correspondence to: Hyman Schipper, Lady Davis Institute, Jewish General Hospital, 3755 Cote St. Catherine Road, Montreal, Quebec, H3T 1E2, Canada. 
a1)

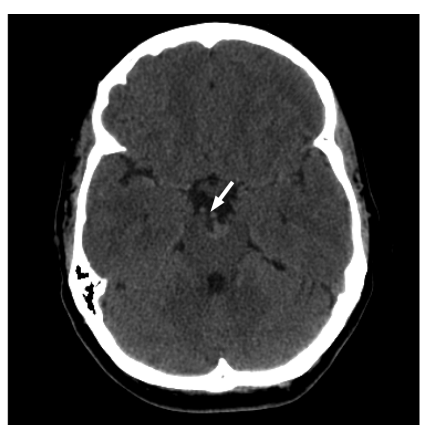

b1)

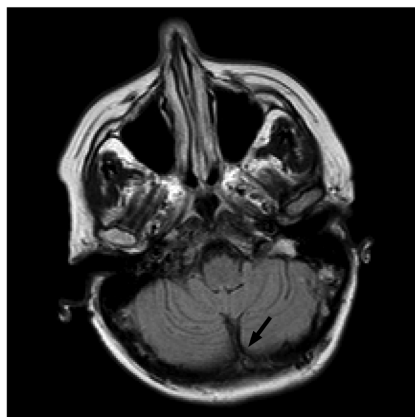

c1)

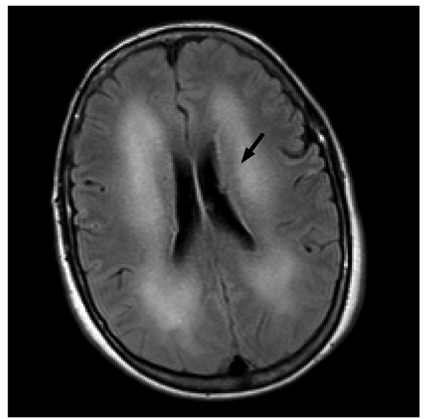

d1)

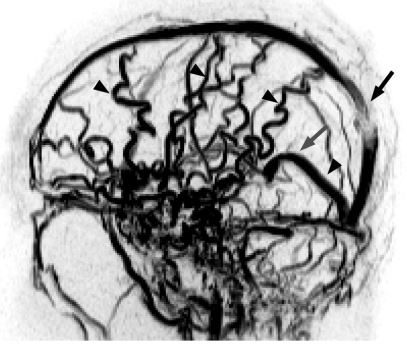

a2)

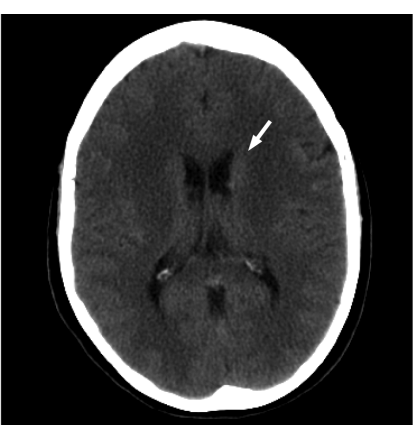

b2)

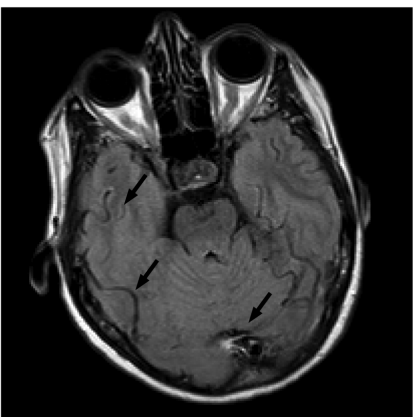

c2)

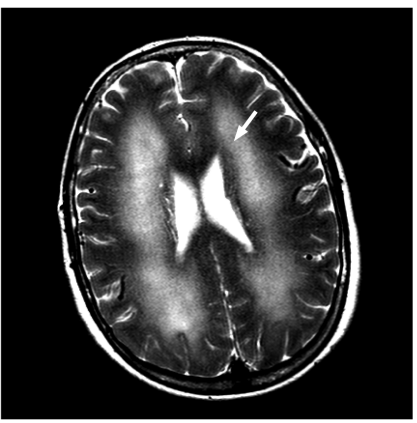

d2)

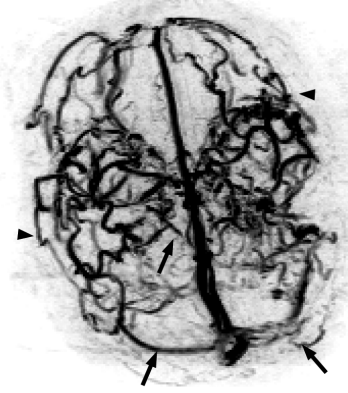

Figure: $C T$ and MR imaging of patient with subacute parkinsonism. a) Axial CT head at the level of midbrain (a1) and lateral ventricle (a2) showing hypodense periventricular white matter and small interpeduncular and periventricular hyperdensities (arrows). b) MR axial FLAIR sequences depicting heterogeneous hyperintense signal of the transverse sinuses (b1, arrow) and congested peripheral veins (b2 arrows). c) MR axial FLAIR (c1) and T2 (c2) images showing diffuse hyperintense signal of the periventricular-subcortical white matter (arrows) and mild sulcal effacement. d): $M R V$ in sagittal (d1) and axial (d2) planes indicating absence of flow in the transverse sinuses, diminished flow in the distal segment of the superior sagittal sinus, distal internal cerebral veins (black arrows) and vein of Galen (grey arrow). There is severe congestion of cortical and subcortical veins and straight sinus (arrowheads).

Parkinsonism of cerebrovascular etiology usually occurs in the context of diffuse cerebrovascular arteriosclerosis/ lipohyalinosis or complicating multiple cerebral infarctions or hemorrhages $^{2}$. The most common clinical picture is one of slow and occasionally punctuated disease progression. Subacute parkinsonism, as reported here, may be due to fungal brain abscess, subdural hematoma, and acute intoxication ${ }^{3}$. The occurrence of subacute micrographia, hypophonia, rigidity and postural instability as a manifestation of cerebral venous occlusion is unusual and we are aware of only two similar cases in the literature ${ }^{4,5}$.
This case is also remarkable for the discordance between the relatively preserved functional status of the patient at initial presentation and the extent and severity of the cerebral venous occlusions on neuroimaging. The absence of focal sensorimotor deficits, increased intracranial pressure, depressed consciousness and seizures, symptoms often accompanying acute CVT, suggests an atypically protracted evolution of veno-occlusion. The presence of dilated cortical/subcortical venous channels ("varicose brain") and clot solidification noted at attempted thrombectomy support this formulation. The absence of infarction within the basal ganglia or thalamocortical projections 
suggests that the extrapyramidal signs may have arisen secondary to the diffuse white matter edema implicating these areas or, given the exaggerated primitive reflexes, broad frontal cortical involvement. The venous drainage of the basal ganglia, thalamus and other subcortical brain structures is comprised of subependymal, thalamostriate, septal, Rosenthal's and anterior pontomesencephalic veins. The septal and thalamostriate veins anastamose to form the internal cerebral veins. The latter join with Rosenthal's veins to form the great vein of Galen ${ }^{6}$. Our patient's extrapyramidal symptoms were likely due to edema of the basal ganglia and thalamocortical projections arising from occlusion of the distal internal cerebral veins and marked flow reduction within the vein of Galen, as demonstrated by MRV. Generalized frontal lobe dysfunction has been described as an etiology of acute-onset micrographia ${ }^{7}$. The current case underscores the potential usefulness of MRV in the evaluation of unexplained white matter changes.

Our patient had no known risk factors for CVT (puerperium, hypercoagulable state, malignancy, medications, etc.) as occurs in $\sim 12 \%$ of reported series ${ }^{8}$. Albeit rare, CVT should be considered as a potentially treatable cause of subacute parkinsonism, particularly in the context of worsening headaches and despite the absence of identifiable risk factors.

\section{REFERENCES}

1. Stam J. Thrombosis of the cerebral veins and sinuses. New Eng J Med. 2005;352(17):1791-8.

2. Winikates J, Jankovic J. Clinical correlates of vascular parkinsonism. Arch Neurol. 1999;56(1):98-102.

3. Waters, CH. Structural lesions and parkinsonism. In: Stern MB, Koller WC, editors. Parkinsonian syndromes. New York: Marcel Dekker; 1993. p. 137-55.

4. Lee SH, Chang H, Kim YS. Deep cerebral venous thrombosis showing parkinsonism such as micrographia, hypophonia and bradykinesia. J Korean Neurol Assoc. 2002;20(2):187-90.

5. Murray BJ, Llinas R, Caplan LR, Scammell T, Pascual-Leone A. Cerebral deep venous thrombosis presenting as acute micrographia and hypophonia. Neurology. 2000;54(3):751-3.

6. Bradley W, Daroff RB, Genichel G, Jankovic J, editors. Neurology in clinical practice, 5th ed. Philadelphia: ButterworthHeinemann/Elsevier; 2008. p. 656.

7. Nakamura $M$, Hamamoto $M$, Uchida $S$, et al. A case of micrographia after subcortical infarction: possible involvement of frontal lobe function. Eur J Neurol. 2003;10(5):593-6.

8. Ferro JM, Canhao P, Stam J, et al. Prognosis of cerebral vein and dural sinus thrombosis: results of the international study on cerebral vein and dural sinus thrombosis (ISCVT). Stroke. 2004; 35(3):664-70. 